\title{
Therapeutic exercise based on biobehavioral approach for the rehabilitation of a radial nerve injury after surgical removal of a schwannoma: a case report
}

\author{
Ferran Cuenca-Martínez ${ }^{1,2}$, Mónica Grande-Alonso ${ }^{1,2}$, Luis Suso-Martit ${ }^{2,3}$, Roy La Touche ${ }^{1,2 *}$ \\ 'Departamento de Fisioterapia, Centro Superior de Estudios Universitarios La Salle, Universidad Autónoma de Madrid, Madrid, Spain \\ ${ }^{2}$ Motion in Brains Research Group, Institute of Neuroscience and Movement Sciences (INCIMOV), Centro Superior de Estudios Universitarios La Salle, Universidad \\ Autónoma de Madrid, Madrid, Spain \\ ${ }^{3}$ Department of Physiotherapy, Universidad Cardenal Herrera-CEU, CEU Universities, Valencia, Spain
}

Schwannoma is a benign tumor located in the Schwann cells of the nerves. A 36-year-old white man underwent surgery after being diagnosed with radial nerve schwannoma of the upper right extremity between the triceps brachii muscle and brachioradialis muscle, after which a complete axonotmesis of the aforementioned nerve trunk occurred. The symptomatology presented by the patient included a loss of both motor and sensory function of the fingers, hand, and right forearm. The treatment was a biobehavioral physiotherapy approach that included therapeutic exercise, neuro-orthopedic manual physical therapy, therapeutic education, and sensitivity retraining. The biobehavioral approach in physiotherapy includes a multimodal approach that encompasses biological aspects, along with cognitive-evaluating and motivational-affective components. Two weekly sessions were held for 6 months, resulting in a total of 48 sessions. Using multimodal physiotherapy based on a biobehavioral approach, after 6 months of treatment, the symptoms improved. The motor function of the upper limb improved considerably due to the recovery of increased activity of the superficial musculature dependent on the radial nerve. However, the functions dependent on deep forearm and hand musculature recovered to a lesser extent. At the sensory level, the retraining of sensitivity allowed the patient to regain an important sensitivity. This case report explains the treatment used and the findings obtained, given a large percentage of the functionality that had been lost was recovered.

Keywords: Biobehavioral physiotherapy, Radial nerve schwannoma, Surgery repair, Neurodynamic mobilization, Therapeutic exercise

\section{INTRODUCTION}

Tumor pathology is uncommon in nerve tissues of the upper extremities. Within this unusual pathological presentation, schwannoma is the tumor that occurs most frequently (Kang et al., 2000). Although cases of tumor cell expansion have been described, usually located in the Schwann cells of these nerves, schwannoma is a benign tumor (Kang et al., 2000).

The usual treatment performed to remove the neoplasm is surgery, which aims to remove the tumor cells present in the Schwann cells while attempting to maintain the maximum integrity of the affected nerve, thus obtaining a good prognosis in these cases. However, surgery is not always optimal because structural injury to nerve tissue has sometimes been reported after the surgery, requiring repair (Kang et al., 2000). With regard to surgical treatment, sometimes the tumor contains a capsule that can encompass all, or some nerve fibers with what is usually performed a microsurgery of "shelling out." Afterwards, an autologous autograft is usually performed using other peripheral nerves such as the sural nerve or the medial cutaneous arm nerve with the aim of restoring the continuity of the nerves (Kang et al., 2000).

These kind of complex situations require a biobehavioral approach, because the influence of both the affective-emotional such as kinesiophobia, depressive symptoms or anxiety and the cogni-
*Corresponding author: Roy La Touche (ib https://orcid.org/0000-0001-6379-6155 Facultad de Ciencias de la Salud, Centro Superior de Estudios Universitarios La Salle, Calle la Salle, no 10, Madrid 28023, Spain

E-mail: roylatouche@yahoo.es

Received: June 5, 2019 / Accepted: July 11, 2019
This is an Open Access article distributed under the terms of the Creative Commons Attribution Non-Commercial License (http://creativecommons.org/licenses/by-nc/4.0/) which permits unrestricted non-commercial use, distribution, and reproduction in any medium, provided the original work is properly cited. 
tive-evaluating variables such a catastrophic thoughts, bad expectations, low self-efficacy beliefs, or erroneous beliefs could act as a barrier to patient recovery (Cuenca-Martínez et al., 2018; Gil-Martinez et al., 2018; Turk, 2003; Turk and Okifuji, 2002). The biobehavioral approach aims to improve the patient's motivation, change their beliefs and misconceptions, increase the locus of internal control, and maintain adherence to treatment (Beltran-Alacreu et al., 2015a; Turk, 2003). Beltran-Alacreu et al. (2015a) found that a biobehavioral-based multimodal physical treatment produced a substantial reduction in pain intensity, pain catastrophizing, disability and the impact of headaches in a patient with chronic tension-type headache. In addition, Grande-Alonso et al. (2019) recently showed that physiotherapy based on a biobehavioral approach, with or without orthopedic manual physical therapy, was effective in the treatment of nonspecific chronic low back pain until at least 3 months after receiving the intervention. Similar results were also obtained by Beltran-Alacreu et al. (2015b).

This case study describes the evaluation and development of a multimodal physiotherapy treatment based on a biobehavioral approach in the rehabilitation of a radial nerve injury after surgical removal of a schwannoma. This is the first case study assessing a biobehavioral physiotherapy treatment in a patient with radial nerve schwannoma. The authors thought this approach was necessary because the patient was referred from several care centers without obtaining significant improvements. The treatment the patient previously received was a classic physiotherapy treatment based on electrotherapy and massage (usual care). It is therefore that the purpose of this study was to evaluate and treat a patient with a nerve injury through a biobehavioral approach based on the patient-centered biopsychosocial model.

\section{MATERIALS AND METHODS}

\section{Case history}

The patient was a 36-year-old white man (weight, $70 \mathrm{~kg}$; height,
$178 \mathrm{~cm}$ ) who underwent surgery after being diagnosed with radial nerve schwannoma of the upper right extremity between the triceps brachii muscle and the brachioradialis muscle, after which a complete axonotmesis of the aforementioned nerve trunk occurred. The axonotmesis was later repaired by a bifascicular autograft of the sural nerve. A calendar of the patient's previous history is shown in Appendix 1.

The main symptomatology presented by the patient during the initial interview was a loss of both motor and sensory function of the fingers, hand, and right forearm, together with the presence of burning pain in the area of the forearm and a sense of tightness. The patient underwent a nerve conduction study at 3 and 6 months after the second surgery, in which the results of complete axonotmesis were found, with no evidence of ongoing reinnervation.

\section{Clinical findings}

In relation to the physical therapy evaluation, the abolition of the motor response was observed in the form of loss of muscular mass of the upper right extremity. The burning pain sensation was located on the dorsal side of the forearm.

With regard to range of motion (ROM), a significant loss of active ROM was observed, given evident denervation of the musculature attributed to the radial nerve. The ROM was decreased in all movements of the forearm, wrist and fingers with the exception of the elbow pronation movement. A decrease in active ROM was found in wrist extension, wrist flexion, wrist abduction, wrist adduction, elbow supination, thumb abduction, thumb extension and fingers (second, third, fourth, and fifth finger) extension, which were in a fallen position. All baseline measurements (T0) of the active ROM in the affected upper limb are found in the Table 1. The ROM was measured through a mobile application consisting of a digital goniometer, whereas the movements of pronation and supination were measured with a hand goniometer (Pourahmadi et al., 2017).

In terms of diagnostic tests, a neurodynamic test of the radial

Table 1. Baseline and follow-up measurements of the active range of motion in the affected upper limb from T0 to T4

\begin{tabular}{|c|c|c|c|c|c|c|c|c|}
\hline Measure & $\begin{array}{l}\text { Wrist extension } \\
\left.\text { (H-ROM: } 70^{\circ}\right)\end{array}$ & $\begin{array}{l}\text { Wrist flexion } \\
\left(\text { H-ROM: } 91^{\circ}\right)\end{array}$ & $\begin{array}{l}\text { Elbow supination } \\
\left(\mathrm{H}-\mathrm{ROM}: 87^{\circ}\right)\end{array}$ & $\begin{array}{l}\text { Thumb abduction } \\
\left.\text { (H-ROM: } 63^{\circ}\right)\end{array}$ & $\begin{array}{c}\text { Thumb extension } \\
\left.\text { (H-ROM: } 26^{\circ}\right)\end{array}$ & $\begin{array}{l}\text { Fingers extension } \\
\left(\mathrm{H}-\mathrm{ROM}: 20^{\circ}\right)\end{array}$ & $\begin{array}{c}\text { Radial deviation } \\
\left(\mathrm{H}-\mathrm{ROM}: 22^{\circ}\right)\end{array}$ & $\begin{array}{l}\text { Ulnar deviation } \\
\left.\text { (H-ROM: } 35^{\circ}\right)\end{array}$ \\
\hline TO & $5^{\circ}$ & $50^{\circ}$ & $10^{\circ}$ & $0^{\circ}$ & $0^{\circ}$ & $-13^{\circ}$ & $8^{\circ}$ & $20^{\circ}$ \\
\hline $\mathrm{T} 1$ & $37^{\circ}$ & $77^{\circ}$ & $46^{\circ}$ & $4^{\circ}$ & $0^{\circ}$ & $-8^{\circ}$ & $8^{\circ}$ & $27^{\circ}$ \\
\hline $\mathrm{T} 2$ & $59^{\circ}$ & $84^{\circ}$ & $71^{\circ}$ & $9^{\circ}$ & $6^{\circ}$ & $-2^{\circ}$ & $10^{\circ}$ & $29^{\circ}$ \\
\hline T3 & $62^{\circ}$ & $85^{\circ}$ & $82^{\circ}$ & $10^{\circ}$ & $12^{\circ}$ & $2^{\circ}$ & $10^{\circ}$ & $30^{\circ}$ \\
\hline T4 & $64^{\circ}$ & $85^{\circ}$ & $83^{\circ}$ & $11^{\circ}$ & $13^{\circ}$ & $4^{\circ}$ & $11^{\circ}$ & $31^{\circ}$ \\
\hline
\end{tabular}

H-ROM, healthy upper limb range of motion; T0, baseline measurement; $\mathrm{T}$, measurement at the 2nd month of treatment; $\mathrm{T} 2$, measurement at the 4th month of treatment; $T 3$, measurement at the 6 th month of treatment; $\mathrm{T} 4$, measurement at a month after treatment completion. 
Table 2. Baseline and follow-up measurements of the psychosocial and somatosensory factors from $\mathrm{TO}$ to $\mathrm{T} 4$

\begin{tabular}{lccccc}
\hline Measure & T0 & T1 & T2 & T3 & T4 \\
\hline Month & 0 & 2nd & 4 th & 6 th & 7th \\
PCS (0-52) & 31 & 7 & 3 & 3 & 1 \\
HADS (0-21/0-21) & $16 / 15$ & $12 / 9$ & $8 / 4$ & $6 / 3$ & $5 / 3$ \\
QuickDASH (0-100) & 77.27 & 65.90 & 47.72 & 40.90 & 36.36 \\
NPRS (0-10) & 3 & 1 & 0 & 0 & 0 \\
Two-points DT-A (cm) & - & 7 & 6 & 5 & 5 \\
Two-points DT-H (cm) & 3 & 3 & 3 & 3 & 3 \\
\hline
\end{tabular}

PCS, pain catastrophizing scale; HADS, hospital anxiety and depression scale; QuickDASH, disability of the arm, shoulder, and hand; NPRS, numeric pain rating scale; Two-points DT, 2-point discrimination test; $A$, affected; $H$, healthy.

nerve (ULNT 2B) was performed and very low mechanosensitivity was found. The neurodynamic tests on the median nerve (ULNT 1) and ulnar nerve (ULNT 3) showed no relevant findings. In the somatosensory assessment, the 2-point discrimination test was evaluated in the sensitive region corresponding to the radial nerve, both on the healthy side and on the affected side, with an esthesiometer (Nolan, 1982). The main finding was that no response was found in the 2-point discrimination test in the sensitive territory corresponding to the radial nerve of the affected upper limb. The minimum distance where the healthy upper limb could discriminate was $3 \mathrm{~cm}$ (Table 2).

Finally, a set of self-report questionnaires was given to the patient concerning psychosocial issues, pain, function, and disability: the Spanish version of the pain catastrophizing scale (PCS) to assess the degree of pain catastrophizing, the pain intensity was measured using a numeric pain rating scale (NPRS), the Spanish version of the Hospital Anxiety and Depression Scale, and the Spanish version of QuickDASH (disability of the arm, shoulder, and hand) to evaluate the symptoms and physical disabilities of the patient.

The Spanish version of the PCS assesses the degree of pain catastrophizing. The PCS has 13 items and a 3 -factor structure of rumination, magnification, and helplessness. The highest score on this questionnaire is 52 . Higher scores indicate higher levels of catastrophic thoughts (García-Campayo et al., 2008).

Pain intensity was measured using a NPRS. The NPRS is a segmented numerical version of the analogue visual scale consisting of 11 points, and it ranges from 0 representing "no pain" to 10 representing "worst pain imaginable" (Ferraz et al., 1990).

The Spanish version of hospital anxiety and depression scale (HADS) consist of a 14-item instrument with a 4-item Likert scale that measures from 0 to 3 . This instrument has two sub- scales consisting of seven items each. Both the anxiety and depression subscales have a maximum score of 21 points. Even if it's the same questionnaire, the subscales work separately. Higher scores on each subscale indicate higher levels of depressive symptoms and anxiety (Quintana et al., 2003).

The Spanish version of QuickDASH evaluates the symptoms and physical disabilities of the patient. This version has 11 questions with five alternatives and a maximum score of five for each question. The QuickDASH questionnaire score has a maximum score of 100. Higher scores indicate greater dysfunctionality of the upper limb (Hervás et al., 2006).

\section{Therapeutic intervention}

Two weekly sessions were held for 6 months, resulting in a total of 48 sessions. The biobehavioral physiotherapy approach included therapeutic education, neuro-orthopedic manual physical therapy, therapeutic exercise, and sensitivity retraining. It also encompassed various techniques in neuro-training, such as laterality training, action observation training, kinesthetic motor imagery, and visual mirror feedback. All treatments had a total duration of 30 min spread over 12 sessions. Appendix 2 shows the therapeutic intervention carried put.

\section{RESULTS}

The patient received a total of 48 sessions, each consisting of 30 $\min$ in duration. Five measurements were taken: the first was basal (T0), the second took place 2 months after starting the treatment (T1), the third at the 4th month of treatment (T2), the fourth at the end of treatment (T3), and the fifth a month after treatment completion (T4). The results obtained are presented in the result tables (Tables 1, 2).

The motor function of the upper limb improved considerably due to the recovery of increased activity of the superficial musculature dependent on the radial nerve (brachioradialis, extensor carpi radialis longus, extensor carpi radialis brevis, and elbow supinator muscles). The patient reached $85^{\circ}$ of active wrist flexion, $64^{\circ}$ of active wrist extension, and the total degrees of wrist abduction and adduction was $42^{\circ}$. However, the functions dependent on deep forearm and hand musculature recovered to a lesser extent. At the sensory level, the retraining of sensitivity allowed the patient to regain an important sensitivity such as the ability to respond to an alarm signal (protective function). Nevertheless, this somatosensory recovery was not complete because the subject showed that the minimum distance to discriminate 2 points was 
$5 \mathrm{~cm}$ in the affected upper limb, compared to $3 \mathrm{~cm}$ in the healthy upper limb.

\section{DISCUSSION}

This case report presents a detailed description of the evaluation and the partially successful evolution of an adult male patient with a complete axonotmesis in the radial nerve which was later repaired by a bifascicular autograft of the sural nerve who was treated with physiotherapy based on a biobehavioral approach. The motor and the sensory functions were partially recovered oneand-a-half years after the repair surgery was undertaken. These functions remained completely nonresponsive after surgery, as shown both in the postintervention measurements and in a nerve conduction study.

The brachioradialis, extensor carpi radialis longus, extensor carpi radialis brevis, and supinator muscles function were recovered practically in its entirety, whereas the intermediate and deep musculature recovered only partially (extensor digitorum, extensor digiti minimi, abductor pollicis longus, extensor pollicis, extensor pollicis longus, extensor pollicis brevis, and extensor indicis). Based on the study of Ryu et al. (1991), the most clinically relevant finding was that $40^{\circ}$ of wrist extension, $40^{\circ}$ of wrist flexion, and a total of $40^{\circ}$ of radial-ulnar deviation is needed to perform a majority of the activities of daily living. These results were achieved in the present case study. These improvements were objectified through the QuickDASH questionnaire, which showed a substantial improvement after the intervention.

Neural mobilization, which has shown to promote neural regeneration after peripheral neurogenic injury, was key in the treatment, as were the techniques aimed at provoking cortical reorganization by improving the motor function (e.g., motor imaging and visual mirror feedback) (da Silva et al., 2015). The study conducted by Villafañe et al. (2013), found similar results to those obtained in the present study, where a program with manual therapy and neurodynamic mobilization improved the function of a patient with peroneal nerve paralysis.

At a sensory level, sensitivity retraining allowed the patient to recover some important sensitivity, such as the ability to respond to an alarm signal. The patient had previously suffered burns in the previous rehabilitation centers due to anesthesia in the affected area. Therapeutic education made it possible to reduce the influence of psychosocial variables while improving adherence to treatment, increasing self-efficacy, and improving the motivational aspect. This could be observed through the obtained scores in the self-reported HADS and PCS questionnaires, where their influence practically ceased after the intervention. Therapeutic education has shown similar improvements within a multimodal physiotherapy program in different clinical entities (Beltran-Alacreu et al., 2015b; Kindelan-Calvo et al., 2014). The lack of scientific evidence on a biobehavioral approach for this pathology does not allow us to compare our results with other authors. However, Engquist et al. (2013), using a multimodal physiotherapy treatment based on the biobehavioral approach, showed positive long-term results in patients with cervical radiculopathy.

Clinically, a multimodal physiotherapy based on a biobehavioral approach seems to offer solutions in the biological sphere, but also in cognitive-emotional aspects widely present in complex patients. The use of sensorimotor neurotraining tools may be interesting in patients who have suffered a prolonged disuse of some body area. Therapeutic education should be used by clinicians to improve adherence to treatment, as well as to improve the negative influence of some psychosocial variables such as catastrophic thinking, low self-efficacy, poor expectations, erroneous beliefs, etc. Finally, the neurodynamic technique may be an interesting tool for problems related to altered mechanosensitivity of a peripheral nerve although further research is needed to clarify some aspects.

The present study presents several limitations that should be taken into account. First, since a nerve conduction study could not be performed (at postintervention), there are only indirect evidences of reinnervation of the radial nerve-dependent musculature. The increase of the active ROM and the improvement of the functionality of the affected upper limb are considered as such indirect evidences. Second, it would have been interesting to collect data on the grip strength of the hand in order to compare the results before and after the intervention. Third, changes in cortical reorganization caused by different sensorimotor neurotraining tools such as kinesthetic motor imagery, action observation training or visual mirror feedback must be considered as a hypothesis because no neuroimaging technique confirming this speculation was used in the present case study. In addition, it is important to stress the fact that there were so many types of interventions. When this occurs, it is difficult to establish attributions about which intervention contributed the most to the patient's improvements. Finally, due to the fact that it is a case study, it is impossible to establish comparisons. It is possible that the patient's natural history could have influenced the patient's improvements; however, the authors believe there is strong evidence to think that the treatment had a significant impact on the patient's recovery. 


\section{CONFLICT OF INTEREST}

No potential conflict of interest relevant to this article was reported.

\section{ACKNOWLEDGMENTS}

The authors thank the Instituto de Rehabilitación Funcional (IRF) La Salle and the Centro Superior de Estudios Universitarios (CSEU) La Salle for making this study possible.

\section{REFERENCES}

Anema JR, Steenstra IA, Bongers PM, de Vet HC, Knol DL, Loisel P, van Mechelen W. Multidisciplinary rehabilitation for subacute low back pain: graded activity or workplace intervention or both? A randomized controlled trial. Spine (Phila Pa 1976) 2007;32:291-298.

Beltran-Alacreu H, López-de-Uralde-Villanueva I, Fernández-Carnero J, La Touche R. Manual therapy, therapeutic patient education, and therapeutic exercise, an effective multimodal treatment of nonspecific chronic neck pain: a randomized controlled trial. Am J Phys Med Rehabil 2015a;94(10 Suppl 1):887-897.

Beltran-Alacreu H, Lopez-de-Uralde-Villanueva I, La Touche R. Multimodal physiotherapy based on a biobehavioral approach as a treatment for chronic tension-type headache: a case report. Anesth Pain Med 2015b;5:e32697.

Cuenca-Martínez F, Cortés-Amador S, Espí-López GV. Effectiveness of classic physical therapy proposals for chronic non-specific low back pain: a literature review. Phys Ther Res 2018;21:16-22.

da Silva JT, Santos FM, Giardini AC, Martins Dde O, de Oliveira ME, Ciena AP, Gutierrez VP, Watanabe IS, Britto LR, Chacur M. Neural mobilization promotes nerve regeneration by nerve growth factor and myelin protein zero increased after sciatic nerve injury. Growth Factors 2015;33:8-13.

Engquist M, Löfgren H, Öberg B, Holtz A, Peolsson A, Söderlund A, Vavruch L, Lind B. Surgery versus nonsurgical treatment of cervical radiculopathy: a prospective, randomized study comparing surgery plus physiotherapy with physiotherapy alone with a 2-year follow-up. Spine (Phila Pa 1976) 2013;38:1715-1722.

Ferraz MB, Quaresma MR, Aquino LR, Atra E, Tugwell P, Goldsmith CH. Reliability of pain scales in the assessment of literate and illiterate patients with rheumatoid arthritis. J Rheumatol 1990;17:1022-1024.

García-Campayo J, Rodero B, Alda M, Sobradiel N, Montero J, Moreno S. Validation of the Spanish version of the Pain Catastrophizing Scale in fibromyalgia. Med Clin (Barc) 2008;131:487-492.
Gil-Martínez A, Paris-Alemany A, López-de-Uralde-Villanueva I, La Touche R. Management of pain in patients with temporomandibular disorder (TMD): challenges and solutions. J Pain Res 2018;11:571-587.

Grande-Alonso M, Suso-Martí L, Cuenca-Martínez F, Pardo-Montero J, Gil-Martínez A, La Touche R. Physiotherapy based on a biobehavioral approach with or without orthopedic manual physical therapy in the treatment of nonspecific chronic low back pain: a randomized controlled trial. Pain Med 2019 May 7 [Epub]. pii: pnz093. https://doi. org/10.1093/pm/pnz093.

Hervás MT, Navarro Collado MJ, Peiró S, Rodrigo Pérez JL, López Matéu P, Martínez Tello I. Spanish version of the DASH questionnaire. Crosscultural adaptation, reliability, validity and responsiveness. Med Clin (Barc) 2006;127:441-447.

Kang HJ, Shin SJ, Kang ES. Schwannomas of the upper extremity. J Hand Surg Br 2000;25:604-607.

Kindelan-Calvo P, Gil-Martínez A, Paris-Alemany A, Pardo-Montero J, Muñoz-García D, Angulo-Díaz-Parreño S, La Touche R. Effectiveness of therapeutic patient education for adults with migraine. A systematic review and meta-analysis of randomized controlled trials. Pain Med 2014;15:1619-1636.

Linder M, Michaelson P, Röijezon U. Laterality judgments in people with low back pain--A cross-sectional observational and test-retest reliability study. Man Ther 2016;21:128-133.

Lindström I, Ohlund C, Eek C, Wallin L, Peterson LE, Fordyce WE, Nachemson AL. The effect of graded activity on patients with subacute low back pain: a randomized prospective clinical study with an operant-conditioning behavioral approach. Phys Ther 1992;72:279-290.

López-de-Uralde-Villanueva I, Muñoz-García D, Gil-Martínez A, PardoMontero J, Muñoz-Plata R, Angulo-Díaz-Parreño S, Gómez-Martínez M, La Touche R. A systematic review and meta-analysis on the effectiveness of graded activity and graded exposure for chronic nonspecific low back pain. Pain Med 2016;17:172-188.

Macedo LG, Latimer J, Maher CG, Hodges PW, McAuley JH, Nicholas MK, Tonkin L, Stanton CJ, Stanton TR, Stafford R. Effect of motor control exercises versus graded activity in patients with chronic nonspecific low back pain: a randomized controlled trial. Phys Ther 2012;92: 363-377.

Nolan MF. Two-point discrimination assessment in the upper limb in young adult men and women. Phys Ther 1982;62:965-969.

Pourahmadi MR, Ebrahimi Takamjani I, Sarrafzadeh J, Bahramian M, Mohseni-Bandpei MA, Rajabzadeh F, Taghipour M. Reliability and concurrent validity of a new iPhone ${ }^{\circledR}$ goniometric application for measuring active wrist range of motion: a cross-sectional study in asymptomatic subjects. J Anat 2017;230:484-495.

Quintana JM, Padierna A, Esteban C, Arostegui I, Bilbao A, Ruiz I. Evalu- 
ation of the psychometric characteristics of the Spanish version of the Hospital Anxiety and Depression Scale. Acta Psychiatr Scand 2003; 107:216-221.

Ryu JY, Cooney WP 3rd, Askew LJ, An KN, Chao EY. Functional ranges of motion of the wrist joint. J Hand Surg Am 1991;16:409-419.

Turk DC. Cognitive-behavioral approach to the treatment of chronic pain patients. Reg Anesth Pain Med 2003;28:573-579.

Turk DC, Okifuji A. Psychological factors in chronic pain: evolution and revolution. J Consult Clin Psychol 2002;70:678-690.

Villafañe JH, Pillastrini P, Borboni A. Manual therapy and neurodynamic mobilization in a patient with peroneal nerve paralysis: a case report. J Chiropr Med 2013;12:176-181. 
Appendix 1. Calendar of the patient's previous history

Date History

24/03/2014 Schwannoma of the radial nerve was diagnosed. A nerve conduction study of the radial neuromuscular system was performed, obtaining a conduction velocity and amplitude of the normal sensory evoked potential, without significant asymmetries with respect to the contralateral side.

25/07/2014 Schwannoma of the radial nerve was surgically removed.

02/12/2014 Nerve conduction studies of the radial, median and ulnar nerves were performed. Studies showed the absence of sensory and motor activity of the neuro-musculoskeletal system of the radial nerve.

25/02/2015 A bifascicular autograft surgery (end-to-end anastomosis) of the Sural nerve was performed.

25/05/2015 The nerve conduction study at 3 months postsurgery was carried out, which concluded: "Radial nerve neuropathy in the degree of complete axonotmesis between the triceps brachii muscle and the brachioradialis muscle."

25/08/2015 Nerve conduction study at 6 months postsurgery: "abolished response of the right radial nerve both at the level of the extensor indicis proprius muscle and of the brachioradialis muscle, concluding in the persistence of the absence of response (both in voluntary activity and in response to proximal stimulation of said nerve trunk) in the right radial musculature indicative of complete axonotmesis or neuronotmesis, without evidence of data suggesting reinnervation in progress"

07/09/2015 A physical therapy rehabilitation treatment was scheduled in a Public Hospital in Madrid. A program of 60 sessions of electrotherapy and massage was carried out without any improvement.

22/07/2016 The patient came to us where the first assessment was made.

25/07/2016 Start the intervention

19/01/2017 Completion of the intervention 
Appendix 2. Therapeutic intervention performed

\begin{tabular}{|c|c|}
\hline Phase & Therapeutic intervention \\
\hline $\begin{array}{l}\text { First phase (1st-12th sessions) } \\
\text { The projected goals were to improve neu- } \\
\text { ral mechanosensitivity, to reduce periph- } \\
\text { eral neurogenic pain and the feeling of } \\
\text { tightness experienced by the patient, and } \\
\text { to diminish the influence of any psycho- } \\
\text { social variables. }\end{array}$ & $\begin{array}{l}\text { Neuro-orthopedic manual physical therapy } \\
\text { - Neural mobilizations of radial nerve ( } 5 \text { sets of } 10 \text { mobilizations) each with a break of } 30 \text { sec per set at a rate of } 1 \mathrm{~Hz} \text {. } \\
\text { - Neural automobilizations of radial nerve at home between } 3 \text { and } 5 \text { times a day at a rate of } 10-12 \text { repetitions per set. } \\
\text { Biobehavioral strategies } \\
\text { - Therapeutic education: enhancing the influence of psychosocial variables, modifying thoughts and misconceptions, and } \\
\text { improving self-efficacy and coping strategies ( } 7 \text { sessions of } 15 \text { min). } \\
\text { Sensory-motor neurotraining: One type of sensorimotor neurotraining is discrimination of laterality. Laterality judgment is } \\
\text { defined as the capacity to analyze and identify a body part when it is presented apart from a global image. } \\
\text { - Hand laterality recognition training: Recognize online is an online app designed to assess the ability to perform laterality } \\
\text { judgements. This application displays different right/left images and it accurately measures the speed and accuracy of } \\
\text { making left-/right-hand discrimination judgements of each image. This app has been developed and released by the Neuro } \\
\text { Orthopaedic Institute Group, and provides the option to vary the number of images and the length of time the user has to } \\
\text { view each image. Recognize online was used to carry out sensorimotor retraining with images of the hand through the } \\
\text { "vanilla" option (Linder et al., 2016). There were } 10 \text { trials per session during this phase, each lasting approximately } 45 \text { sec } \\
\text { (12 sessions). }\end{array}$ \\
\hline
\end{tabular}

\section{Second phase (13th-40th sessions)}

During this phase, the level of progression achieved during the previous phase was pursued.

Third phase (41th-48th sessions)

\section{Neuro-orthopedic manual physical therapy (progression)}

- Neural mobilizations of radial nerve+neck lateral gliding.

Sensory-motor neurotraining (progression)

- Motor Imagery was first performed visually, in third person, and afterward, it was enacted in first person using kinesthetic as well as visual input in conjunction with a context.

- Visual mirror feedback was employed. Initially, it was conducted only in a visual manner, without performing the gesture, and later the gesture of the right extremity was added. It was also combined with thermal sensitivity training of the prototype and epicritical touch.

\section{Neuro-orthopedic manual physical therapy (progression)}

- Neurodynamic mobilizations progressed to tensioners performing 4 sets of 15 repetitions, at a frequency of $2 \mathrm{~Hz}$.

Sensory-motor neurotraining (progression)

- We provided action observation training with gestures and hand movements in video format.

Biobehavioral strategies: included a graded exercise intervention and therapeutic education. Graded exercise is a cognitive behavioral approach that consists of three phases: measuring functional capacity, educating in the workplace and providing an individual program of submaximal exercise that is gradually increased. It is therefore that the dosage of the fiscal year is carried out through quotas (López-de-Uralde-Villanueva et al., 2016). It is therefore that graded exercise is based on (a) treatment goals focused on functional exercises; and (b) operant conditioning behavioral principles and improving exercise and activity tolerance using a quota system instead of pain abatement (López-de-Uralde-Villanueva et al., 2016).

- Graded exercise; a primary goal of the program was to increase activity tolerance by performing individualized and submaximal exercises reinforcing wellness behavior. The program was based on activities that the patient identified as problematic and that he could not perform. The patient received daily quotas and was instructed to only perform the agreed amount, not less or more (Anema et al., 2007; Lindström et al., 1992; Macedo et al., 2012). The exercises carried out were: strength training, cardiovascular training and progression to functional activities ( 9 sessions of 20 min plus exercises at home). This exercise model implemented was similar to the one conducted by Macedo et al. (2012).

- Therapeutic education: concepts as well as the activities recommended to perform at home were reviewed to reinforce them. 